\title{
Ion beam induced current analysis in GaN microwires
}

\author{
Dirkjan Verheij ${ }^{1,2,1}$, Marco Peres ${ }^{2}$, Susana Cardoso ${ }^{1}$, Luís Cerqueira Alves ${ }^{3}$, Eduardo Alves $^{2}$, \\ Cristophe Durand ${ }^{4}$, Joël Eymery ${ }^{5}$, Jorge Fernandes ${ }^{6}$, and Katharina Lorenz ${ }^{1,2}$
}

\author{
${ }^{1}$ Instituto de Engenharia de Sistemas e Computadores - Microsistemas e Nanotecnologia (INESC- \\ MN), Rua Alves Redol 9, 1000-029 Lisboa, Portugal \\ ${ }^{2}$ IPFN, Instituto Superior Técnico (IST), Campus Tecnológico e Nuclear, Estrada Nacional 10, 2695 - \\ 066 Bobadela LRS, Portugal \\ ${ }^{3} \mathrm{C} 2 \mathrm{TN}$, Instituto Superior Técnico (IST), Campus Tecnológico e Nuclear, Estrada Nacional 10, 2695- \\ 066 Bobadela LRS, Portugal \\ ${ }^{4}$ CEA INAC-Pheliqs-NPS, Université Grenoble Alpes, Grenoble, France \\ ${ }^{5}$ CEA INAC-MEM-NRS, Université Grenoble Alpes, Grenoble, France \\ ${ }^{6}$ Instituto de Engenharia de Sistemas e Computadores - Investigação e Desenvolvimento (INESC-ID), \\ Rua Alves Redol 9, 1000-029, Lisboa, Portugal
}

\begin{abstract}
GaN is a wide bandgap semiconductor which is expected to withstand high radiation doses. Consequently, it is considered a promising material for new generation particle detectors in radiation related applications. We report on the fabrication and electrical characterization under proton irradiation of single microwire sensors based on a back-toback Schottky contact configuration. The microwires are grown by metalorganic vapor phase epitaxy and processed into sensors by using optical lithography on dispersed wires. We investigate the impact of the contacts and the semiconductor bulk on the ion beam induced current (IBIC) by irradiating specific areas of the sensor and simultaneously measuring the change in conductivity. We observed that the contribution of the excess charge carriers generated in the depletion regions formed at the contact interfaces is of low influence when compared to the excess charge carriers generated in the microwire bulk.
\end{abstract}

\section{Introduction}

The development of radiation resistant electronics and sensors is fundamental to enable technology that can endure extreme harsh environments such as those encountered in space or nuclear facilities. One measure to improve the lifetime of devices is using radiation resistant materials like $\mathrm{GaN}$. GaN is a wide bandgap semiconductor with a good radiation hardness due to its high average displacement energy values, the energy required to displace an atom from its lattice position [1]. Additionally, the radiation defects are mobile even at low temperature, leading to dynamic annealing during the irradiation [2].

1 Corresponding author: dirkjanverheij@ctn.tecnico.ulisboa.pt 
Nonetheless, despite being a matured technique, the heteroepitaxial growth of $2 \mathrm{D}$ films still presents issues, caused mainly by the lattice mismatch between $\mathrm{GaN}$ and the typical growth substrates, which leads to the presence of a high density of dislocations. Exchanging 2D technology with nano- and microwire processing solved this problem to a great extent. Due to the free lateral surface of the wire, the strain caused by the mismatch is relaxed without creating dislocations or the dislocations are bent to the sidewall surface, very close to the bottom of the wire [3].

The detection of ionizing radiation using GaN microwires was reported previously [4]. During proton irradiation a change in conductivity was measured and transient measurements at fixed bias showed a relative current increase of approximately $40 \%$. A strong degradation of the sensor, rendered as an increase of the resistance, was however also observed when the fluence surpassed $1 \times 10^{16}$ protons $/ \mathrm{cm}^{2}$. Also, the presence of a persistent ionocurrent was observed with decay transients of the same order of magnitude as the persistent photocurrent (i.e. current induced by UV light) measured prior to proton irradiation. Persistent ionocurrents were previously observed as well in $\mathrm{ZnO}$ nanowires [5]. In $\mathrm{GaN}$ wire structures, the persistent photocurrent is attributed to surface recombination. Due to the surface band bending, holes are pushed to surface and in order to recombine, electrons must overcome the corresponding potential barrier [6].

In the present study, single GaN microwire devices are processed by depositing contacts on their extremities. The creation of potential barriers at the interface between the contact metal and the semiconductor leads to a configuration consisting of back-to-back Schottky contacts. We can thus divide the device in three main areas, the forward biased Schottky contact, the reverse biased Schottky contact and the central area of the microwire. The aim of this study is to analyze each of these areas regarding their influence in the electrical current induced by the ion beam irradiation.

\section{Experimental methods}

GaN microwires were grown on a c-plane sapphire substrate by catalyst-free metal organic vapor phase epitaxy (MOVPE) [7]. To initiate vertical growth, a silane flux needs to be added during the first stage of the growth which leads to a very high incorporation of silicon donor atoms. When the silane flux is turned off halfway the growth process, the $\mathrm{Si}$ incorporation decreases. Consequently, the bottom part of the wire is heavily doped $\left(10^{20}\right.$ $\mathrm{cm}^{-3)}$, while the upper part of the wire is moderately doped $\left(10^{18} \mathrm{~cm}^{-3}\right)[8,9]$. More details on the growth process and characterization of the as-grown microwires can be found in [7]. The length of the wires ranges from 20-25 $\mu \mathrm{m}$ whereas the diameter lies between 1-2 $\mu \mathrm{m}$. Fig. 1a) shows an $\mathrm{SEM}$ image of a single microwire deposited on a $\mathrm{Si} / \mathrm{SiO}_{2}$ substrate.

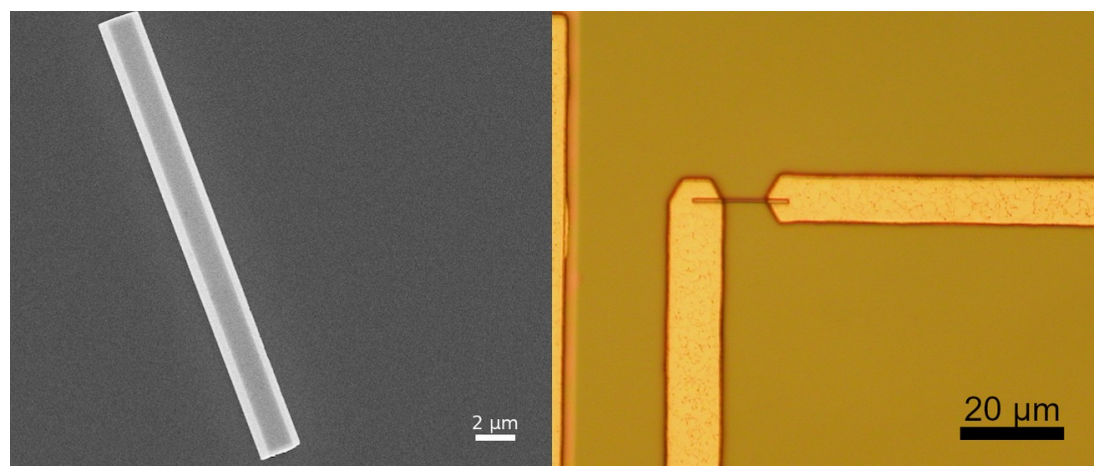

Fig. 1. a) SEM image of a deposited microwire. b) Optical microscopy image of a single wire radiation sensors after processing. 
The radiation sensors were fabricated in the form of single microwire devices, with contacts on the extremities of the wires in a similar process as described previously [4]. Prior to the microwire deposition on the device substrate, a metallic pattern containing alignment markers was defined using a standard optical lithography process and a layer of $\mathrm{SiO}_{2}$ was deposited to avoid conduction between the wires and the substrate. The wires were then dispersed on the $\mathrm{SiO}_{2}$ using a wet process. A small piece of the wire growth substrate was placed in an Eppendorf tube together with a small volume of isopropyl alcohol (IPA). The tube was placed in an ultrasonic bath for 60 seconds which leads to detachment of the wires from their growth substrate. Finally, a dozen of droplets of the suspension were placed on the device substrate leading, after cleaning the surface with IPA and deionized water, to a uniform distribution of wires. Using the alignment markers, the position of the microwires were defined and metallic Ti/Au (300/4000 $\AA$ ) contacts were deposited using sputtering. An optical microscope image of a final device is shown in Fig. $1(b)$.

For the irradiation, hydrogen atoms are ionized and accelerated by a Van de Graaff accelerator to an energy of $2 \mathrm{MeV}$ and directed towards a nuclear microbeam setup [10], where the beam is focused to an area of 3 by $4 \mu \mathrm{m}^{2}$. Taking into account that the beam current is around $70 \mathrm{pA}$, we have an average ion flux of approximately $3 \times 10^{15}$ protons $\mathrm{cm}^{-2}$ $\mathrm{s}^{-1}$. The small size of the beam spot combined with the ability to control its position allow the irradiation of specific areas of the device. The electrical properties of the radiation detectors are analyzed prior, during and after the proton irradiation using an Agilent B1500A semiconductor device analyzer. All I-V curves were measured in the dark.

\section{Results}

Fig. 2 shows the initial current-voltage (I-V) characteristic at room temperature before irradiation. The I-V curve exhibits a non-linear and asymmetric behavior, where the conductivity is higher for positive bias. The non-linearity is a direct consequence of the back-to-back Schottky barriers present at the contacts. The asymmetry is also expected due to the inhomogeneous doping at both extremities of the wire. Since the thickness of the potential barrier is inversely proportional to the square root of the doping concentration [11], the barrier located at the heavily doped extremity has a lower thickness.

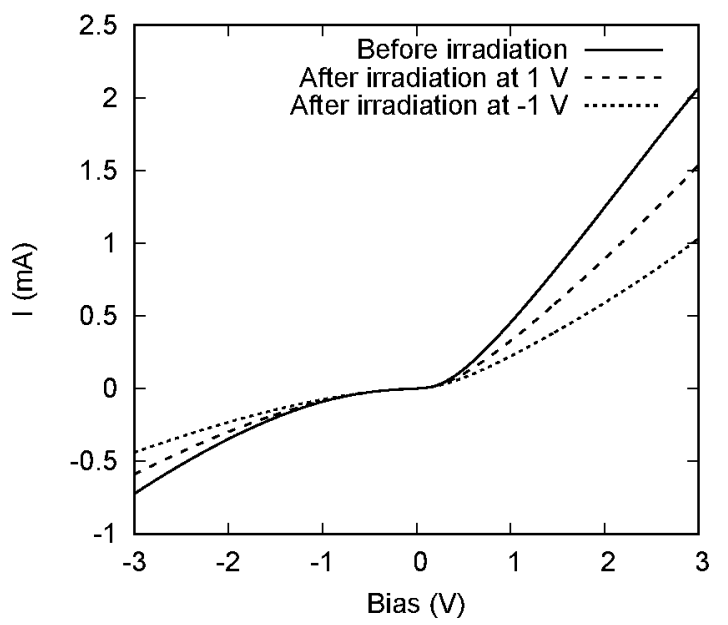

Fig. 2. Experimental I-V characteristics obtained before irradiating the device (solid line) and after irradiating the device (dashed lines). The medium dashed line corresponds to an I-V curve taken after the irradiation at $1 \mathrm{~V}$ and the short dashed line ta an $\mathrm{I}-\mathrm{V}$ curve measured after the irradiation at $-1 \mathrm{~V}$. 
Therefore, when this contact in reverse bias (corresponding to a positive bias in the graph) the tunneling of electrons through the barrier is enhanced, leading to a higher conductivity and a more linear characteristic. When applying a linear regression to the linear range $(1.5$ to $3.0 \mathrm{~V}$ ) we can extract a resistance of $1207.3 \pm 0.5 \Omega$.

For the irradiation analysis, the sample is placed on a sample holder inside the microbeam chamber, which is evacuated to a pressure of $10^{-5}-10^{-6} \mathrm{mbar}$. With the aid of proton induced X-ray emission (PIXE) maps the beam is aligned with the sample. Seven areas were defined along the longitudinal direction of the microwire. Area I is located away from the wire, areas II and III are located on the contact with the heavily doped extremity, areas IV and V correspond to the central area of the wire and, finally, areas VI and VII refer to the irradiation at the moderately doped extremity. The beam is placed on each of these areas for a short time instant ( $\sim 2$ seconds) in order to minimize the damage and consequent conductivity loss induced by the irradiation. At the same time the current is being measured at a fixed bias. The measurement was done for a bias of +1 Volt, with the contact at the higher doped extremity in reverse bias and repeated for a bias of -1 Volt where the higher doped extremity is forward biased. Due to the back-to-back Schottky configuration of the device, when irradiating the sample, there are two possible mechanisms that can generate ion beam induced current (IBIC). The interaction of the proton beam with the semiconductor leads to the generation of a large number of excess charge carriers due to the band to band excitation. These excess charges are free to move in the semiconductor, therefore, the conductivity of the material increases during the proton irradiation. Beyond this mechanism, near the depletion region at the metal-semiconductor junction, free charge carriers created in the space charge region of the device are separated rapidly by the presence of the internal electric field [12]. In our case, we would expect that the reverse biased Schottky barrier would limit current flow without irradiation and a strong increase of current would be expected upon irradiation. If there was a strong influence of this latter effect, we would observe it when irradiating the areas corresponding to contacts that are reverse biased, as observed by Hirsch et al. when performing IBIC measurement on a $(\mathrm{Al}, \mathrm{Ga}) \mathrm{N}$ MSM structure [13]. However, as is shown in fig. 3, the results obtained at positive and negative bias follow the same general behavior. If the contacts would have a significant contribution to the ionocurrent, for positive bias we would see high signals for areas II and III and low signals for areas VI and VII. For negative bias the opposite would happen. Instead, we observe that the IBIC is independent on the polarity of the applied bias.
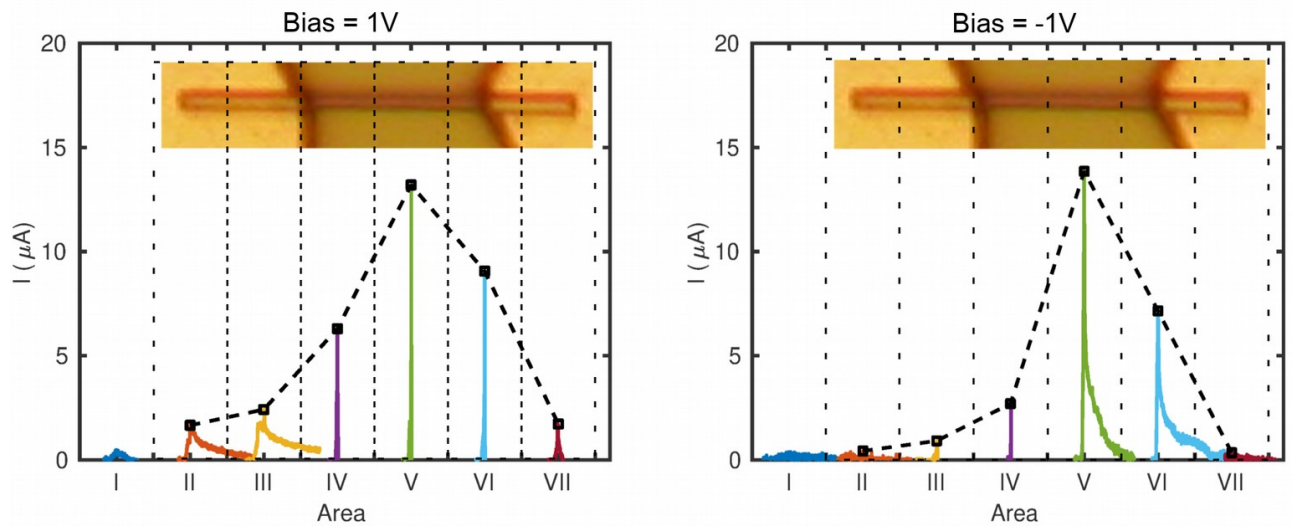

Fig. 3. Ion beam induced current measurement when irradiating the different areas of the microwire, at a bias of $1 \mathrm{~V}$ (left) and at a bias of $-1 \mathrm{~V}$ (right). The dashed lines indicate which area corresponds to each peak. For both positive and negative bias it is clear that the highest IBIC is measured when irradiating area V. Note that for each measurement the background current was subtracted. 
This indicates the biggest share of the IBIC is due to free electrons excited to the conduction band in the semiconductor bulk (note that holes in GaN have lower mobility and thus contribute less to the IBIC). A possible explanation for this observation is that, due to the high doping levels, the depletion region, where the free carriers are separated by the internal electric field occurs is too small to provide a significant contribution.

At this moment it is not clear why the IBIC signal is lower in the regions closer to the extremities of the microwires. We note that the alignment of the beam with the microwire is challenging and irradiation may not be completely homogeneous and that the beam intensity can present some instabilities over time. However, assuming that we are irradiating the area corresponding to the extremity of the microwire covered with the metal electrode, the volume of created charge carriers and corresponding IBIC should be of the same magnitude as in the central areas of the wire. Thus, although the experimental results seem to imply that the there is no Schottky effect contribution to the increase of the IBIC, the metal-semiconductor interface seems active in suppressing it. It is possible that this is caused by the different nature of surface defects at the metal-semiconductor interface when compared to the defects present at the semiconductor surface not covered by the metal. The lack of control over the position is however a limitation of the experimental setup making it hard to determine the precise location of the irradiation. Additionally, as mentioned above, the spot size of the beam has an area of 3 by $4 \mu \mathrm{m}^{2}$, which is roughly twice the diameter of the microwire. Consequently, the resolution is relatively small compared to, for example, electron beam induced current (EBIC) measurements [14].

It is also worth looking at the decay of the conductivity due to the damage created by the irradiation. Fig. 2 shows the I-V curve measured after each experiment. We can see that the current drops consistently. By performing a linear regression to linear section of the I-V curves we obtain resistances of $1596 \pm 2 \Omega$ and $2374 \pm 5 \Omega$, corresponding to the situation after irradiation with a fluence of $(5 \pm 2) \times 10^{16}$ protons $/ \mathrm{cm}^{2}$ (after irradiation at positive bias) and a fluence of $(9 \pm 3) \times 10^{16}$ protons $/ \mathrm{cm}^{2}$ (after irradiation at negative bias) respectively.

\section{Conclusions}

In this work the mechanisms of IBIC were studied taking advantage of the characteristics of the microbeam setup allowing irradiation of distinct areas of the device. Although there is some contribution of the Schottky contacts to the I-V curves, the overall influence of the built-in electric field at the Schottky contact on the IBIC signal is small, however we observe a strong suppression of the IBIC in these regions. Although we obtain good stability and reproducible measurements, we observed a decay in conductivity due to the induced irradiation damage. This can have influence on the transient behavior as the creation of traps influences the generation and recombination mechanisms.

In this case however, due to the similar dimensions of the beam and the sample, the resolution of the measurements is not good enough to study the generation processes in depth, nonetheless, it shows there is potential to perform position dependent irradiation measurements for larger size samples.

We acknowledge funding by FCT and FEDER (PTDC/CTM-CTM/28011/2017, LISBOA-01-0145FEDER-028011)

\section{References}

[1] S.J. Pearton, R. Deist, F. Ren, L. Liu, A.Y. Polyakov, J. Kim, Journal of Vacuum Science and Technology A 31, 050801 (2013) 
[2] K. Lorenz, M. Peres, N. Franco, J. G. Marques, S. M. C. Miranda, S. Magalhães, T. Monteiro, W. Wesch, E. Alves, E. Wendler, Proc. SPIE 7940 7940, 79400 (2011)

[3] Coulon P M, Mexis M, Teisseire M, Jublot M, Vennéguès P, Leroux M, Zuniga-Perez, J. Appl. Phys. 115, 153504 (2014)

[4] D. Verheij, M. Peres, S. Cardoso, L. C. Alves, E. Alves, C. Durand, J. Eymery, K. Lorenz, J. Phys. D: Appl. Phys. 51, 175105 (2018)

[5] A. Johannes, R. Niepelt, M. Gnauck, C. Ronning, Appl. Phys. Lett. 99, 252105 (2011)

[6] R. Callarco, M. Marso, T. Richter, A. I. Aykanat, R. Meijers, A. v.d. Hart, T. Stoica, H. Lüth, Nano Letters 5, 981 (2005)

[7] R. Koester, J. S. Hwang, C. Durand, D. L. S. Dang, J. Eymery, Nanotechnology 21, 015602 (2010)

[8] P. Tchoulfian, F. Donatini, F. Levy, B. Amstatt, P. Ferret, J. Pernot, Appl. Phys. Lett. 102, 122116 (2013)

[9] P. Tchoulfian, F. Donatini, F. Levy, B. Amstatt, A. Dussaigne, P. Ferret, E. Bustarret, J. Pernot, Appl. Phys. Lett. 103, 202101 (2013)

[10] N. F. Santos, A. J. S. Fernandes, L. C. Alves, N. A. Sobolev, E. Alves, K. Lorenz, F. M. Costa, T. Monteiro, Nucl. Instrum. Methods Physics Res. B 306, 195 (2013)

[11] S. M. Sze and K. N. Kwok, Eds., Physics of Semiconductor Devices, 3rd ed. New Jersey: John Whiley \& Sons, 2007.

[12] Q. Xu, P. Mulligan, J. Wang, W. Chuirazzi, L. Cao, Nucl. Instrum. Methods Phys. Res. A 849, 11 (2017)

[13] L. Hirsch, P. Tardy, G. Wantz, N. Huby, P. Moretto, L. Serani, F. Natali, B. Damilano, J.Y. Duboz, J.L. Reverchon, Nucl. Instrum. Methods Phys. Res. B 240, 265 (2005)

[14] P. Lavenus, A. Messanvi, L. Rigutti, A. De Luna Bugallo, H. Zhang, F. Bayle, F. H. Julien, C. Durand, M. Tchernycheva, Nanotechnology 25, 255201 (2014) 